\title{
Stellar interactions in dense and sparse star clusters
}

\author{
C. Olczak ${ }^{1}$, S. Pfalzner ${ }^{1}$, and A. Eckart ${ }^{1,2}$ \\ 1 I. Physikalisches Institut, Universität zu Köln, Zülpicher Str.77, 50937 Köln, Germany \\ e-mail: olczak@ph1.uni-koeln.de \\ 2 Max-Planck-Institut für Radioastronomie, Auf dem Hügel 69, 53121 Bonn, Germany
}

Received 5 June 2009 / Accepted 2 November 2009

\begin{abstract}
Context. Stellar encounters potentially affect the evolution of the protoplanetary discs in the Orion Nebula Cluster (ONC). However, the role of encounters in other cluster environments is less known.

Aims. We investigate the effect of the encounter-induced disc-mass loss in different cluster environments.

Methods. Starting from an ONC-like cluster we vary the cluster size and density to determine the correlation of the collision time scale and disc-mass loss. We use the NBODY6++ code to model the dynamics of these clusters and analyse the disc-mass loss due to encounters.

Results. We find that the encounter rate strongly depends on the cluster density but remains rather unaffected by the size of the stellar population. This dependency translates directly into the effect on the encounter-induced disc-mass loss. The essential outcome of the simulations are: i) even in clusters of four times lower density than the ONC, the effect of encounters is still apparent; ii) the density of the ONC itself marks a threshold: in less dense and less massive clusters it is the massive stars that dominate the encounter-induced disc-mass loss, whereas in denser and more massive clusters the low-mass stars play the major role for the disc-mass removal. Conclusions. It seems that in the central regions of young dense star clusters - the common sites of star formation - stellar encounters do affect the evolution of the protoplanetary discs. With higher cluster density low-mass stars become more heavily involved in this process. These results can also be applied to extreme stellar systems: in the case of the Arches cluster one would expect stellar encounters to destroy the discs of most of the low- and high-mass stars in several hundred thousand years, whereas intermediate mass stars are able to retain their discs to some extent even under these harsh environmental conditions.
\end{abstract}

Key words. stellar dynamics - methods: numerical - stars: pre-main sequence - circumstellar matter

\section{Introduction}

According to current knowledge, planetary systems form from the accretion discs around young stars. These young stars are in most cases not isolated, but are part of a cluster (e.g. Lada \& Lada 2003). Densities in these cluster environments vary considerably, spanning a range of $10 \mathrm{pc}^{-3}$ (e.g. $\eta$ Chameleontis) to $10^{6} \mathrm{pc}^{-3}$ (e.g. Arches Cluster). Though it is known that discs disperse over time (Haisch et al. 2001; Hillenbrand 2002; Sicilia-Aguilar et al. 2006; Currie et al. 2008) and that in dense clusters $\left(n \gtrsim 10^{3} \mathrm{pc}^{-3}\right)$ the disc frequency seems to be lower in the core (e.g. Balog et al. 2007), it is an open question as to how far interactions with the surrounding stars influence the planet formation in clusters of different densities.

Two major mechanisms are potentially able to strongly affect the evolution of protoplanetary discs and planets in a cluster environment: photoevaporation and gravitational interactions. Photoevaporation causes the heating and evaporation of disc matter by the intense UV radiation from massive stars. First models of photoevaporation were developed by Johnstone et al. (1998) and Störzer \& Hollenbach (1999) (see also references in Hollenbach et al. 2000) and have been much improved in the past years (Clarke et al. 2001; Matsuyama et al. 2003; Johnstone et al. 2004; Alexander et al. 2005, 2006; Ercolano et al. 2008; Gorti \& Hollenbach 2009; Drake et al. 2009). Gravitational interactions are another important effect on the population of stars, discs and planets in a cluster environment. An encounter between a circumstellar disc and a nearby passing star can lead to a significant loss of mass and angular momentum from the disc. While such isolated encounters have been studied in a large variety (Heller 1993; Clarke \& Pringle 1993; Ostriker 1994; Heller 1995; Hall et al. 1996; Hall 1997; Pfalzner 2004; Pfalzner et al. 2005; Moeckel \& Bally 2006, 2007b; Kley et al. 2008), only a few numerical studies have directly investigated the effect of stellar encounters on circumstellar discs in a dense cluster environment (Scally \& Clarke 2001; Adams et al. 2006).

It has been shown only recently by numerical simulations that stellar encounters do have an effect on the discs surrounding stars in a young dense cluster (Olczak et al. 2006; Pfalzner et al. 2006; Pfalzner 2006; Moeckel \& Bally 2006, 2007a,b; Pfalzner \& Olczak 2007a,b; see also the review by Zinnecker \& Yorke 2007). The massive stars in the centre of such a stellar cluster act as gravitational foci for the lower mass stars (Pfalzner et al. 2006). Discs are most affected when the masses of the stars involved in an encounter are unequal (Olczak et al. 2006; Moeckel \& Bally 2007b), so it is the massive stars which dominate the encounter-induced disc-mass loss in the young dense clusters (Olczak et al. 2006). Observational evidence for this effect has been found by Olczak et al. (2008).

The numerical results obtained in our previous investigations are based on a dynamical model of the Orion Nebula Cluster (ONC) - one of the observationally most intensively studied young star cluster. It was demonstrated that in the ONC stellar 
encounters can have a significant impact on the evolution of the young stars and their surrounding discs (Pfalzner 2006; Olczak et al. 2006; Pfalzner et al. 2006; Pfalzner \& Olczak 2007a; Olczak et al. 2008; Pfalzner et al. 2008). However, investigating one model star cluster is not sufficient to draw general conclusions - in fact, one could not answer questions as: How would things change in a denser cluster? Would a higher density inevitably imply that stellar encounters play a more important role in the star - and planet formation process? And what would the situation be like in more massive clusters? Would the larger number of stars - in particular massive stars - play a role?

Conclusive answers to these questions demand further numerical investigations covering a larger parameter space in cluster parameters. Here we realise this by modelling clusters as scaled versions of the standard ONC model with varying stellar numbers and sizes. The focus of this investigation is on the encounter-induced disc-mass loss. Throughout this work we assume that initially all stars are surrounded by protoplanetary discs. This is justified by observations that reveal disc fractions of nearly $100 \%$ in very young star clusters (e.g. Haisch et al. 2000; Lada et al. 2000; Haisch et al. 2001; Hillenbrand 2005). In Sect. 2 we outline briefly the observationally determined basic properties of the ONC, which serves as a reference model for the other cluster models. The computational method is described in Sect. 3 and the construction of the numerical models is outlined in Sect. 4. Afterwards we present results from a numerical approach to this problem in Sect. 5 and compare with analytical estimates in Sect. 6. The conclusion and discussion mark the last section of this paper.

\section{Structure and dynamics of the ONC}

The ONC is a rich stellar cluster with about 4000 members with masses of $m \geq 0.08 M_{\odot}$ and a radial extension of $\sim 2.5 \mathrm{pc}$ (Hillenbrand \& Hartmann 1998; Hillenbrand \& Carpenter 2000). Most of the objects are $\mathrm{T}$ Tauri stars. The mean stellar mass is about $\bar{m} \approx 0.5 M_{\odot}$ and the half-mass radius $R_{\mathrm{hm}} \approx 1 \mathrm{pc}$ (Hillenbrand \& Hartmann 1998). Recent studies of the stellar mass distribution (Hillenbrand \& Carpenter 2000; Luhman et al. 2000; Muench et al. 2002; Slesnick et al. 2004) reveal no significant deviation from the generalised IMF of Kroupa (2002),

$\xi(m)= \begin{cases}m^{-1.3}, & 0.08 \leq m / M_{\odot}<0.50, \\ m^{-2.3}, & 0.50 \leq m / M_{\odot}<1.00, \\ m^{-2.3}, & 1.00 \leq m / M_{\odot}<\infty .\end{cases}$

The mean age of the whole cluster has been estimated to be $t_{\mathrm{ONC}} \approx 1 \mathrm{Myr}$, although a significant age-spread of the individual stars is evident (Hillenbrand 1997; Palla \& Stahler 2000).

The density and velocity distribution of the ONC resembles an isothermal sphere. The central number density $\rho_{\text {core }}$ in the inner $0.053 \mathrm{pc}$ reaches $4.7 \times 10^{4} \mathrm{pc}^{-3}$ (McCaughrean et al. 2002) and makes the ONC the densest nearby $(<1 \mathrm{kpc})$ young stellar cluster. The dense inner part of the ONC, also known as the Trapezium Cluster (TC), is characterised by $R_{\mathrm{TC}} \lesssim 0.3 \mathrm{pc}$ and $N_{\mathrm{TC}} \approx 500$, or $\rho_{\mathrm{TC}} \approx 4 \times 10^{3} \mathrm{pc}^{-3}$.

In the most detailed study on circumstellar discs in the Trapezium Cluster, Lada et al. (2000) found a fraction of 80-85\% discs among the stellar population from the $L$-band excess. This agrees with an earlier investigation of the complete ONC in which Hillenbrand et al. (1998) report a disc fraction of $50-90 \%$ (though relying only on $I_{\mathrm{C}}-K$ colors) and justifies the assumption of a $100 \%$ primordial disc fraction in the simulations presented here.
We now describe the construction of the numerical cluster models that have been used in our simulations.

\section{Computational method}

The basic dynamical model of the ONC used here is described in Olczak et al. (2006), but we include several extensions discussed in Pfalzner \& Olczak (2007b). We summarise the main aspects of our model: the initial stellar population consists of $4000 \mathrm{mem}-$ bers with masses of between $0.08 M_{\odot}$ and $50 M_{\odot}$ sampled from the standard Kroupa IMF (see Eq. (1)). The system is initially in virial equilibrium,

$Q_{\mathrm{vir}}=\frac{R_{\mathrm{hm}} \sigma^{2}}{2 G M}=0.5$,

where $R_{\mathrm{hm}}$ is the half-mass radius of the cluster, $\sigma$ the velocity dispersion, $M$ the total mass, and $G$ the gravitational constant. It is characterised by a radial density profile, $\rho \propto r^{-2}$, with a central density $\sim 4 \times 10^{4} \mathrm{pc}^{-3}$. We adopt a Maxwellian velocity distribution as would be expected from the theory of star cluster formation (e.g. Clarke et al. 2000) and which is roughly in agreement with observations of the star-forming regions (e.g. Falgarone \& Phillips 1990; Miesch \& Scalo 1995). We use here just a singlestar model only and do not include the effect of gas expulsion or stellar evolution. All simulations have been performed with the direct $N$-body code NBODY6++ (Spurzem 1999; Aarseth 2003).

For the generation of star cluster models in the present investigation the initial radial density profile has been slightly modified. In a first-order approximation, the isothermal sphere, $\rho(r) \propto r^{-2}$, represents the projected density distribution of the ONC, yet a flattening in the core, $\Sigma(r) \propto r^{-0.5}, 0<r \leq R_{\text {core }}$, $R_{\text {core }} \approx 0.2 \mathrm{pc}$, is observed (Scally et al. 2005). Validating the initial setup by means of the best reproduction of the current projected density distribution of the $\mathrm{ONC}$ after a simulation time of $1 \mathrm{Myr}$, the evaluation of numerous initial configurations led to the following best estimate of the initial three-dimensional density distribution:

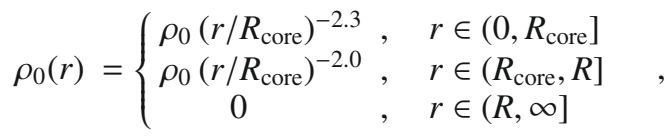

where $\rho_{0}=3.1 \times 10^{3} \mathrm{stars} \mathrm{pc}^{-3}, R_{\text {core }}=0.2 \mathrm{pc}$, and $R=2.5 \mathrm{pc}$.

Moreover, the generation of the high-mass end of the mass function has been modified. In the case of the ONC the upper mass was chosen to be $50 M_{\odot}$, because this value corresponds to the mass of the most massive stellar system in the ONC. However, stars with larger masses are expected to form in more massive clusters (Oey \& Clarke 2005; Weidner \& Kroupa 2006). Thus in the framework of this numerical investigation the upper mass limit has been set to the currently accepted fundamental upper mass limit, $m_{\max }=150 M_{\odot}$ (Figer 2005; Oey \& Clarke 2005; Koen 2006; Weidner \& Kroupa 2006; Maíz Apellániz et al. 2007; Zinnecker \& Yorke 2007).

The choice of a fixed upper mass limit, though in disagreement with the well-established non-trivial correlation of the mass of a star cluster and its most massive member (e.g. Larson 2003; Weidner \& Kroupa 2006; Maschberger \& Clarke 2008), was motivated by the fact that the exact relation is not known. However, a comparison with the "sorted sampling algorithm" of Weidner \& Kroupa (2006) in Table 1 shows that - at least in a statistical sense - the exact prescription for the generation of the maximum stellar mass in a cluster is not as important as it might 
Table 1. Averaged initial parameters of the two families of cluster models.

\begin{tabular}{|c|c|c|c|c|c|c|c|c|c|c|c|}
\hline Family of models & & & sity-scaled & & & & e-scaled & & & & \\
\hline$N$ & model & $\begin{array}{c}R \\
{[\mathrm{pc}]}\end{array}$ & $\begin{array}{c}\rho_{\mathrm{TC}} \\
{\left[10^{3} \mathrm{pc}^{-3}\right]}\end{array}$ & $\begin{array}{c}\sigma_{3 \mathrm{D}} \\
{\left[\mathrm{km} \mathrm{s}^{-1}\right]}\end{array}$ & model & $\begin{array}{c}R \\
{[\mathrm{pc}]}\end{array}$ & $\begin{array}{c}\rho_{\mathrm{TC}} \\
{\left[10^{3} \mathrm{pc}^{-3}\right]}\end{array}$ & $\begin{array}{c}\sigma_{3 \mathrm{D}} \\
{\left[\mathrm{km} \mathrm{s}^{-1}\right]}\end{array}$ & $\begin{array}{l}m_{\max }^{\operatorname{med}} \\
{\left[M_{\odot}\right]}\end{array}$ & $\begin{array}{l}m_{\max }^{\text {sort }} \\
{\left[M_{\odot}\right]}\end{array}$ & $\begin{array}{l}m_{\max }^{\mathrm{obs}} \\
\left.M_{\odot}\right]\end{array}$ \\
\hline 1000 & D0 & 2.50 & 1.3 & 1.15 & S0 & 0.63 & 4.8 & 2.42 & 36 & 32 & $25 \pm 15$ \\
\hline 2000 & D1 & 2.50 & 2.7 & 1.64 & S1 & 1.25 & 5.1 & 2.37 & 52 & 45 & $25 \pm 15$ \\
\hline 4000 & D2 & 2.50 & 5.3 & 2.26 & $\mathrm{~S} 2$ & 2.50 & 5.3 & 2.26 & 79 & 63 & $55 \pm 25$ \\
\hline 8000 & D3 & 2.50 & 10.5 & 3.11 & S3 & 5.00 & 5.3 & 2.13 & 94 & 80 & $75 \pm 25$ \\
\hline 16000 & D4 & 2.50 & 21.1 & 4.34 & S4 & 10.00 & 5.3 & 2.11 & 125 & 112 & $95 \pm 35$ \\
\hline 32000 & D5 & 2.50 & 42.1 & 6.04 & S5 & 20.00 & 5.2 & 2.03 & 137 & 126 & $100 \pm 35$ \\
\hline
\end{tabular}

Note: the first column contains the number of stars, $N$, the next eight columns contain the designation, the cluster size, $R$, the number density in a sphere of a radius of $0.3 \mathrm{pc}, \rho_{\mathrm{TC}}$ (equivalent to the Trapezium Cluster in the ONC, see Sect. 2), and the three-dimensional velocity dispersion, $\sigma_{3 \mathrm{D}}$, for the density-scaled and size-scaled models, respectively. The last three columns denote the median maximum stellar mass in each simulation, $m_{\max }^{\text {med }}$, the median maximum stellar mass for sorted sampling, $m_{\max }^{\text {sort }}$, and estimates from observational data, $m_{\max }^{\text {obs }}$, both taken from Fig. 7 of Weidner \& Kroupa (2006).

seem. The values obtained by random sampling are only slightly larger than those from sorted sampling.

Stellar encounters in dense clusters can lead to a significant transport of mass and angular momentum in protoplanetary discs (Olczak et al. 2006; Pfalzner et al. 2006; Pfalzner $\&$ Olczak 2007a). In the present investigation we assume that all discs are of a low mass, i.e. a low mass ratio of disc and central star, $m_{\text {disc }} / m_{\star} \ll 0.1$, which agrees with observations of the ONC (Bally et al. 1998; Williams et al. 2005; Mann \& Williams 2009). We use Eq. (1) from Pfalzner et al. (2006) to keep track of the relative disc-mass loss of each star-disc system due to encounters. Approaches of stars are only considered to be encounters if the calculated relative disc-mass loss is higher than $3 \%$, corresponding to the $1 \sigma$ error in our simulations of star-disc encounters. In this case the relative disc-mass loss is independent of the disc-mass and depends only on the mass ratio of the interacting stars and the orbital parameters (see Pfalzner et al. 2006). Our estimate of the accumulated disc-mass loss is an upper limit because the underlying formula is only valid for co-planar, prograde encounters, which are the most perturbing. A simplified prescription assigns stars into one of two distinct groups: if the relative disc-mass loss exceeds $90 \%$ of the initial disc mass, stars are marked as "discless"; otherwise they are termed "star-disc systems". As in our previous investigations, the determination of the disc-mass loss involves two different models of the initial distribution of disc sizes: i) scaled disc sizes $r_{\mathrm{d}}$ with $r_{\mathrm{d}}=150 \mathrm{AU} \sqrt{M_{1}}\left[M_{\odot}\right]$, which is equivalent to the assumption of a fixed force at the disc boundary; and ii) equal disc sizes with $r_{\mathrm{d}}=150 \mathrm{AU}$. Whenever results are presented, we will specify which of these two distributions has been used.

\section{Numerical models}

For the present study we have set up a variety of scaled versions of the standard ONC model cluster with varying sizes and stellar numbers. In total, eleven cluster models have been set up (see Table 1). They form two parametric groups, the "densityscaled" (D0-D5) and the "size-scaled" (S0-S5) group, both containing six clusters with stellar numbers of 1000, 2000, 4000, 8000,16000 , and 32000 , respectively. Models D2 and S2 are identical and correspond to the standard ONC model with the adopted higher stellar upper mass limit, $m_{\max }=150 M_{\odot}$. The other ten cluster models have been set up as scaled representations of ONC-like clusters. As in the case of the numerical model of the ONC, for each cluster model a set of simulations has been performed with varying random configurations of positions, velocities, and masses, according to the given distributions, to minimise the effect of statistical uncertainties. For the clusters with 1000, 2000, 4000, 8000, 16000, and 32000 particles, a number of 200,100,100,50,20, and 20 simulations seemed appropriate to provide sufficiently robust results.

It has to be noted that these "artificial" stellar systems are not just theoretical models but have counterparts as well in the observational catalogues of star clusters: the young star cluster NGC 2024 (e.g. Haisch et al. 2000; Bik et al. 2003; Liu et al. 2003 ) is well represented by the 1000 particle model, whereas the 16000 particle model has its counterpart in the massive cluster NGC 3603 (e.g. de Pree et al. 1999; Stolte et al. 2004, 2006).

\section{Density-scaled cluster models}

The six density-scaled cluster models (D0-D5) have been simulated with the same initial size as the $\operatorname{ONC}(R=2.5 \mathrm{pc})$. Due to the adopted number density distribution, which is roughly represented by $\rho(r)=\rho_{0} r^{-2}$, the density of the models scales as the stellar number,

$N=\int_{0}^{R} \rho(r) r^{2} \mathrm{~d} r \mathrm{~d} \Omega \propto \rho_{0} R$,

though for an exact treatment one would have to consider the steeper density profile of the core, $\rho_{\text {core }}(r)=\rho_{\text {core }, 0} r^{-2.3}$,

$N_{\text {core }}=\int_{0}^{R_{\text {core }}} \rho_{\text {core }}(r) r^{2} \mathrm{~d} r \mathrm{~d} \Omega \propto \rho_{\text {core }, 0} R_{\text {core }}^{0.7}$.

However, since the core population is not dominant in terms of the number, the clusters are characterised in good approximation by densities that are $1 / 4,1 / 2,1,2,4$, and 8 times the density of the ONC (at any radius), respectively. These models are used to study the importance of the density for the effect of star-disc encounters in a cluster environment.

\section{Size-scaled cluster models}

Five more cluster models have been simulated with the same initial density as the ONC, but with varying extension. The set of size-scaled cluster models (S0-S5) is used to study the pure effect of the size of the stellar population. Due to the relation (4), the initial size of these clusters scales as the stellar number and was set up with $1 / 4,1 / 2,1,2,4$ and 8 times the initial size of the ONC, respectively.

The initial parameters of the cluster models, for each model averaged over all configurations, are presented in Table 1 . Here 


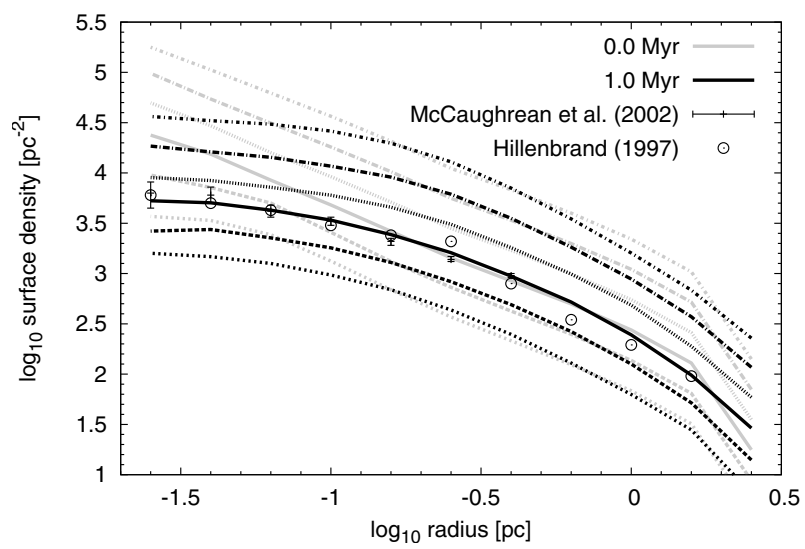

Fig. 1. Projected density profiles of the density-scaled models compared to observational data. The initial profile (grey lines) and the profile at a simulation time of $1 \mathrm{Myr}$ (black lines) are shown. From bottom to top the cluster models D0-D5 are marked in each colour regime by a short-dashed, long-dashed, solid, dotted, dot-long-dashed, and dotshort-dashed line, respectively. The observational data are from a compilation of McCaughrean et al. (2002) and Hillenbrand (1997).

the number density in the Trapezium Cluster, $\rho_{\mathrm{TC}}$, is taken as a reference value for all simulations. As expected, the density scales with the number of stars for the density-scaled models, while it is rather constant for the size-scaled models. The velocity dispersion, which satisfies the relation

$\sigma=\sqrt{\frac{2 G M}{R}} \propto \sqrt{\frac{N}{R}}$

shows the expected scaling of $\sqrt{N}$ for the density-scaled models, and is again roughly constant for the size-scaled models, as was to be expected from $N \propto R$ (Eq. (4)) and the above relation. The reason for the slight increase of the velocity dispersion with a decreasing stellar number for the size-scaled models is the steeper density profile in the cluster core, which becomes more dominant in terms of stellar number with decreasing cluster size. A combination of $N_{\text {core }} \propto R^{0.7}$ from Eq. (5) yields for the above relation roughly $\sigma_{\text {core }} \propto N^{-0.3}$ and thus explains the correlation.

\section{Results of numerical simulations}

In this section the results of the numerical simulations of the two families of cluster models will be presented. A short discussion of the characteristic scaling relations and differences of the cluster dynamics will be followed by a more detailed investigation of the encounter-induced disc-mass loss among the two families of density-scaled and size-scaled cluster models.

\subsection{Cluster dynamics}

In Fig. 1 the evolution of the projected density distribution of the density-scaled models is shown. The shape of the distributions is very similar in all cases. The evolved distributions have nearly identical shapes and are separated by vertical intervals of 0.3 in log-space, which corresponds to the difference of the initial densities by a factor of 2 . Only in the innermost cluster regions slight deviations between the evolved distributions are apparent. These are attributed to the poorer random sampling of the initial particle distribution due to the very steep density profile, $\rho(r) \propto r^{-2.3}$, as is evident from the larger scatter among the initial profiles. However, after 1 Myr these deviations are smoothed out to a large degree.

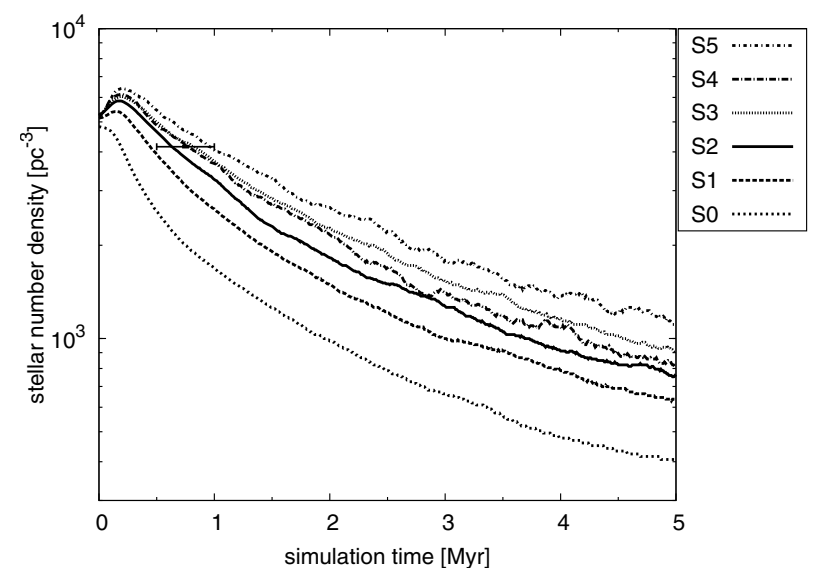

Fig. 2. Time evolution of stellar densities $\rho_{\mathrm{TC}}$ (in a volume of radius $R_{\mathrm{TC}}=0.3 \mathrm{pc}$, see Sect. 2) of the size-scaled cluster models. The cluster models S0-S5 are marked by a short-dashed, long-dashed, solid, dotted, dot-long-dashed, and dot-short-dashed line, respectively. The horizontal error bar marks the corresponding observational estimates for comparison.

Due to the nearly exact qualitative and scaled quantitative evolution of the density-scaled cluster models, it is justified to ascribe differences of the effects of star-disc encounters on the stellar population mainly to one parameter, namely the initial density of the cluster models. Nevertheless, the influence of the particle number has to be considered, too.

The size-scaled cluster models show a different dynamical evolution compared to the density-scaled models. The temporal evolution of the densities $\rho_{\mathrm{TC}}$ in Fig. 2 demonstrates that the clusters evolve on slightly different time scales, where the density declines faster for the less populated clusters S0 and S1. However, the densities of the models S1-S5 differ not much and are consistent with a coeval decline. The evolution of the cluster densities does not - in a first-order approximation - depend on the number of particles, probably with the exception of the model S0. The size-scaled models are thus well suited to the investigation of the effect of the number of cluster stars on star-disc encounters and the corresponding induced disc-mass loss.

\subsection{Encounter dynamics}

We investigate the effect of the cluster density on the encounterinduced disc-mass loss via the evolution of the cluster disc fraction (CDF). The distributions in Fig. 3 show the average fraction of stars that are surrounded by disc material and correspond from top to bottom to clusters with an increasing density. The curves have been smoothed with Bezier curves to provide a clearer view. It is evident that the fraction of destroyed discs in the Trapezium Cluster increases significantly with increasing cluster density. In particular, the effect becomes much stronger for the models D3-D5 with two to eight times the density of the ONC. In the case of the densest model D5, even up to $60 \%$ of the stars in the Trapezium Cluster could have lost their discs after $1 \mathrm{Myr}$ of dynamical evolution. But it is also interesting to note that even in a cluster four times less dense than the ONC (model D0), still 10-15\% of the stars could lose their surrounding discs due to gravitational interactions with cluster members. However, one has to treat these numbers with care due to the partially - significant uncertainties that go into the calculations (see Olczak et al. 2006). Nonetheless, what is more important here - and relies only on the relative quantities - is the fact that 


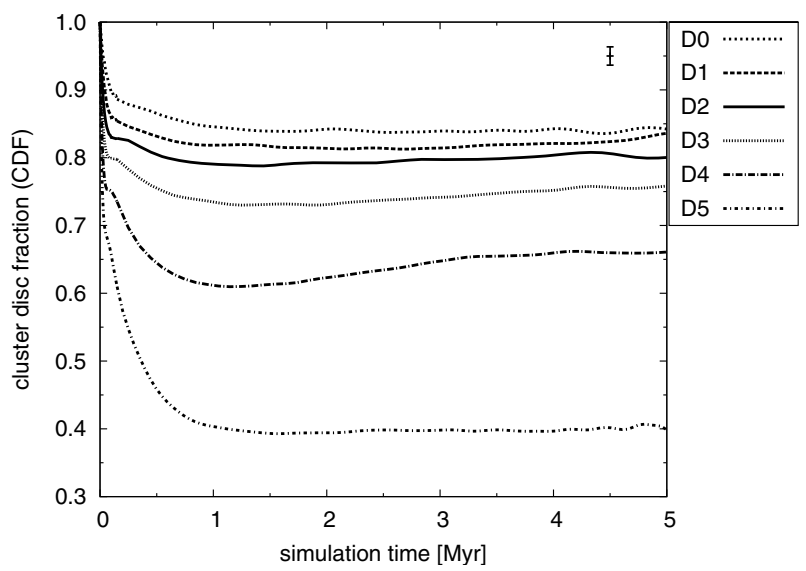

Fig. 3. Time evolution of the cluster disc fraction of the density-scaled models for a region of the size of the Trapezium Cluster $\left(R_{\mathrm{TC}}=0.3 \mathrm{pc}\right)$. The curves have been smoothed by Bezier curves to avoid intersecting lines. Here initially equal disc sizes have been assumed. From top to bottom the cluster models D0-D5 are marked by a short-dashed, longdashed, solid, dotted, dot-long-dashed, and dot-short-dashed line, respectively. The typical error-bar is indicated in the upper right.

the distributions in Fig. 3 are not equidistant but do show larger differences with increasing density.

The impression that the disc fraction increases at later stages of the cluster evolution is solely due to the acceleration of perturbed systems leaving the sampling volume. The escape rate follows the trend of the disc destruction rate with a time delay which is determined by the difference of the crossing time of disc-less stars and star-disc systems in the sampling volume. Hence, the decrease of the disc fraction is followed by an increase and eventually remains constant some time after disc destruction has stopped.

The preferred mass range of stars which become disproportionately strongly involved in perturbing encounters changes significantly between the models D2 and D4. From previous investigations (Olczak et al. 2006; Pfalzner et al. 2006) we already know that in the model D2, representing an ONC-like cluster, the high-mass stars' discs are the ones most affected by encounters. These encounters occur preferentially with low-mass stars due to gravitational focusing. However, in the model cluster D4, a system four times denser, it is the low-mass stars that dominate the disc-mass loss of the cluster population.

We demonstrate this transition by binning the fraction of encounters, adapted to the total number of encounters of each model (so that the integrated surface is unity in each case). Recall that encounters have been defined as flybys causing more than $3 \%$ of disc-mass loss (see Sect. 3). Figure 4 shows for the group of low-mass stars that the dominance of perturbations from much more massive stars in the models D0-D2, represented by the peak at relative perturber masses $\gtrsim 100$, changes dramatically towards encounters of nearly equal-mass stars in the model D4 (with a peak at relative perturber masses $\approx 3$ ). Similarly, the typical geometry of the encounter orbits is subject to a strong transition. As is evident from Fig. 5, most encounters of low-mass stars in the models D0-D2 are parabolic (with an eccentricity $e \approx 1$ ). In contrast, in model D4 these stars usually experience a strongly hyperbolic flyby of a perturber, mostly with an eccentricity $e>10$. Hence, with increasing cluster density, the dominant mode of the star-disc interactions changes towards hyperbolic encounters of low-mass stars among themselves.

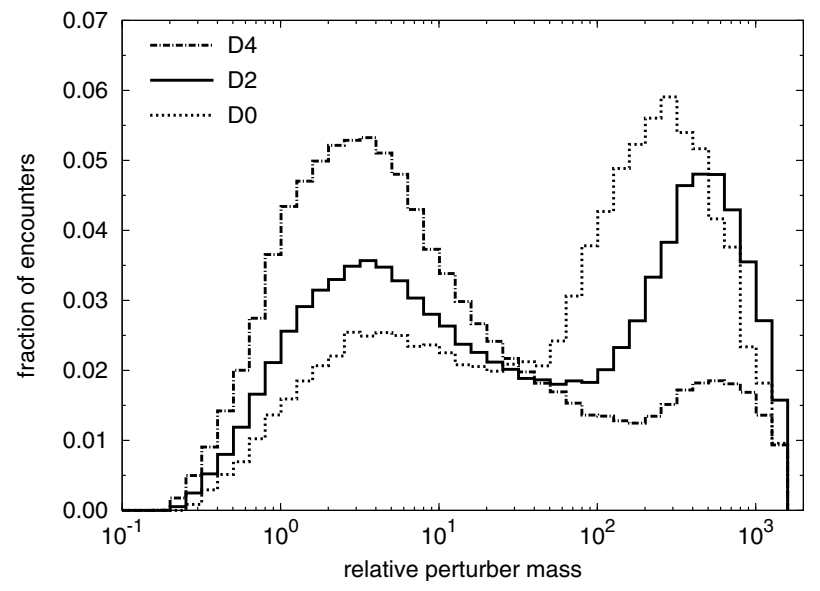

Fig. 4. Comparison of the fraction of encounters as a function of the relative perturber mass, i.e. the mass ratio of perturber and perturbed star, of the group of low-mass stars (see Appendix A for the width of the mass intervals) for the models D0 (dotted), D2 (solid), and D4 (dotlong-dashed), respectively. Here initially equal disc sizes have been assumed.

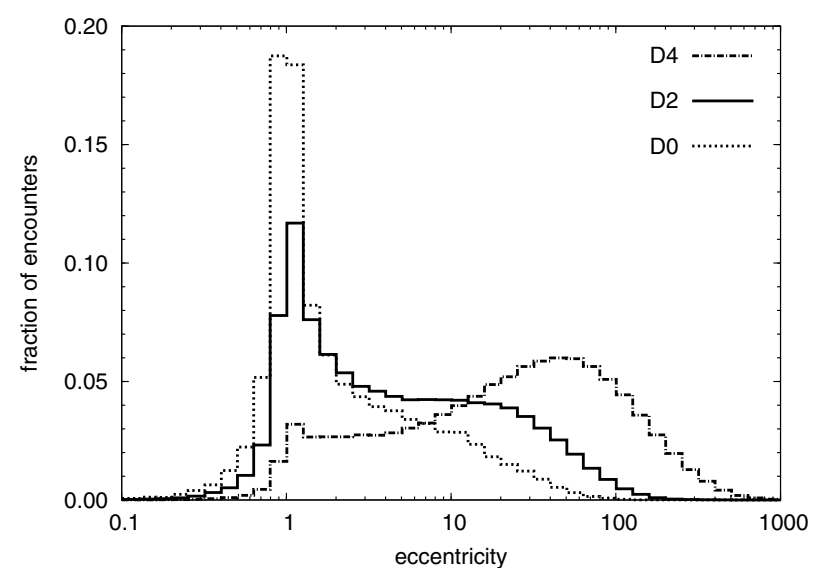

Fig. 5. Comparison of the fraction of encounters as a function of eccentricity of the group of low-mass stars (see Appendix A for the width of the mass intervals) for the models D0 (dotted), D2 (solid), and D4 (dotlong-dashed), respectively. Here initially equal disc sizes have been assumed.

This transition is a consequence of the gravitational focusing by high-mass stars becoming significantly less important among the models D0-D5. Gravitational focusing forces a deflection of the interacting stars onto a less eccentric orbit, effectively increasing the cross-section above geometrical:

$\pi b^{2}=\pi r_{\min }^{2}(1+\Theta)$

where

$\Theta=\frac{v_{\min }^{2}}{\left\langle v_{\text {rel }}\right\rangle^{2}}=\frac{\pi}{16} \frac{v_{\min }^{2}}{\sigma^{2}}$

is the gravitational focusing term or Safronov number, $b$ the impact parameter, $r_{\min }$ the minimum distance, $v_{\min }$ the escape speed at the minimum distance, $\left\langle v_{\text {rel }}\right\rangle$ the mean relative speed, and $\sigma$ the cluster velocity dispersion (one finds $\left\langle v_{\text {rel }}\right\rangle=4 \sigma / \sqrt{\pi}$ for a Maxwellian distribution). Adopting the typical cluster and encounter parameters from Tables 1 and 2 respectively and substituting $v_{\text {min }}=v_{\text {enc }}$ and $r_{\text {min }}=r_{\text {enc }}$, we find that gravitational focusing by low-mass stars is negligible $(\Theta \ll 1)$ in all our cluster models. In contrast, high-mass stars in the model D0 play a substantial role as gravitational foci $(\Theta \approx 120)$, whereas their 
Table 2. Typical stellar encounter parameters.

\begin{tabular}{llllc}
\hline \hline Mass groups & $m_{\star}$ & $r_{\text {enc }}$ & $v_{\text {enc }}$ & $\gamma_{\text {enc }}$ \\
& {$\left[M_{\odot}\right]$} & {$[\mathrm{AU}]$} & {$[\mathrm{AU} / \mathrm{yr}]$} & \\
\hline low mass & $0.1-1$ & $10^{2-3}$ & $\sim 0.3$ & $10^{-1}$ \\
intermediate mass & $1-10$ & $10^{2-2.5}$ & $\sim 2$ & 1 \\
high mass & $10-100$ & $10^{2}$ & $\sim 6$ & 10 \\
\hline
\end{tabular}

Note: the first column denotes the three mass groups, the second column contains the adopted mass ranges, $m_{\star}$, while in the third typical interaction radii, $r_{\mathrm{enc}}$, are listed for each mass group. In the last two columns the resulting "encounter escape speeds", $v_{\text {enc }}$, and the "gravitational focusing parameter", $\gamma_{\mathrm{enc}}$, are noted (see text for definitions of these quantities).

cross section is reduced by more than one order of magnitude in the model D5 $(\Theta \approx 4)$.

In summary, the encounter-induced disc-mass loss in cluster environments of different densities shows two important features: i) low-mass stars lose a larger fraction of their disc material with increasing cluster density; and ii) the discs of the most massive stars are (nearly) completely destroyed; regardless of the density of the cluster environment. The important finding for i) is that the correlation is not linear, but shows a much larger increase for the cluster models with higher densities than model D2, implying that there exists a critical density $\rho_{\text {crit }}$ that marks the onset of a much more destructive effect of star-disc encounters. This critical density seems to be close to the density of the ONC, $\rho_{\text {crit }} \approx \rho_{\text {ONC }}$.

We find that the evolution of the CDF in the Trapezium Cluster region is very similar among the size-scaled models and corresponds to the distribution of the model D2 in Fig. 3. The size-scaled models are obviously of the same importance in their environmental effect on protoplanetary discs despite the slightly different dynamical evolution. The density of the models SO and $\mathrm{S} 1$ decreases faster than for the more massive clusters, even up to a factor of 2 in the case of the model S0 (see Sect. 5.1). Thus one would expect a lower encounter rate in these smaller systems and, consequently, on average a lower disc-mass loss. However, this is obviously not the case and is explained by the fact that, similarly to the finding for the density-scaled models, high-mass stars become less important as gravitational foci for the lowmass stars in clusters with larger stellar populations. Hence in terms of encounter statistics, the lower density of the models S0 and $\mathrm{S} 1$ is compensated by the more frequent interactions of the high-mass stars.

\subsection{Validation of the numerical method}

So far the disc-mass loss has been calculated from the Eq. (1) of Pfalzner et al. (2006) by treating all encounters as parabolic. To account for the lower disc-mass loss in hyperbolic encounters, we have determined a function that quantifies the reduction of the disc-mass loss in dependence of the orbital eccentricity:

$$
\begin{aligned}
\widehat{\Delta m}(e) & =\exp [0.12(e-1)] \\
& \times\{0.83-0.015(e-1)+0.17 \exp [0.1(e-1)]\} .
\end{aligned}
$$

Equation (9) is a fit function to the median distribution of the relative disc-mass loss as a function of eccentricity, adapted to the parabolic case, for all star-disc simulations that have been performed.

As shown in Fig. 6, the number of hyperbolic encounters is significantly reduced if the eccentricity is considered explicitly
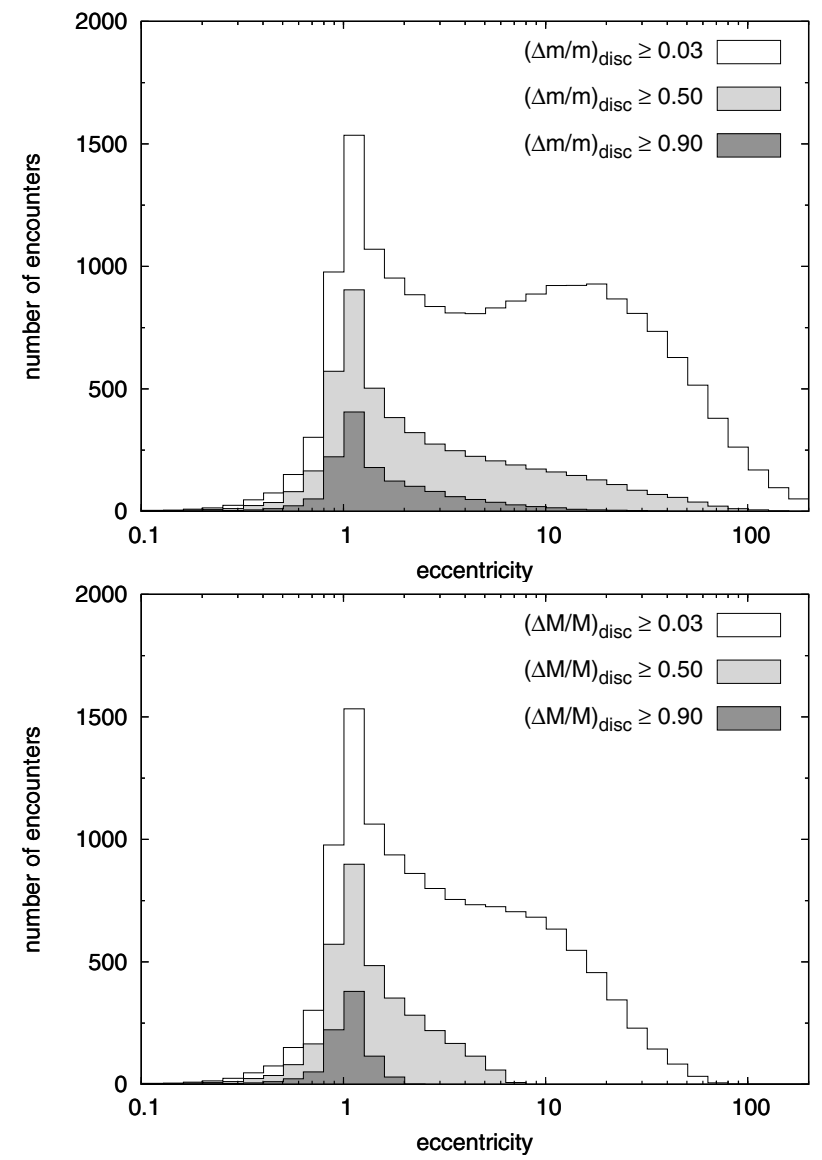

Fig. 6. Number of encounters as a function of eccentricity (logarithmic bins), plotted for three different groups by means of disc-mass loss per encounter. The white surface represents all encounters (i.e. a minimum of 3\% disc-mass loss, cf. Olczak et al. 2006), the light grey surface those that removed at least $50 \%$ of the disc-mass, while the dark grey one stands for the most destructive encounters which caused a discmass loss of at least $90 \%$. The two plots show the distributions for the model D4. Top: disc-mass loss calculated assuming parabolic encounters. Bottom: disc-mass loss calculation corrected for effects of eccentricity by using Eq. (9).

in the calculation of the disc-mass loss using the fit function (9). This is a consequence of our definition of an encounter (see Sect. 3): the fraction of those perturbations which cause a relative disc-mass loss of above $3 \%$ is lower for higher eccentricities, hence the number of eccentric encounters decreases. However, because the fit function represents the median distribution of all simulated star-disc encounters and the effect of strongly perturbing encounters is only weakly dependent on the eccentricity, the number of strongly perturbing encounters in Fig. 6b (light and dark grey surfaces) is underestimated.

It is accordingly the disc-mass loss induced by weak hyperbolic interactions that has been overestimated in the calculations. These events are most numerous in the models D3-D5 with densities of $\rho>\rho_{\text {crit }}$ and result preferentially from interactions of low-mass stars with roughly equal-mass perturbers. Consequently, when considering explicitly the reduced discmass loss in hyperbolic encounters (see Fig. 7), the outstanding role of these dense clusters as environments of huge disc destruction becomes less pronounced (cf. Fig. 3), though the encounterinduced disc-mass loss is still considerably larger compared to the sparser clusters D0-D2.

In Fig. 8 we investigate the effect of the higher upper mass limit on our results as compared to the basic ONC model. It 


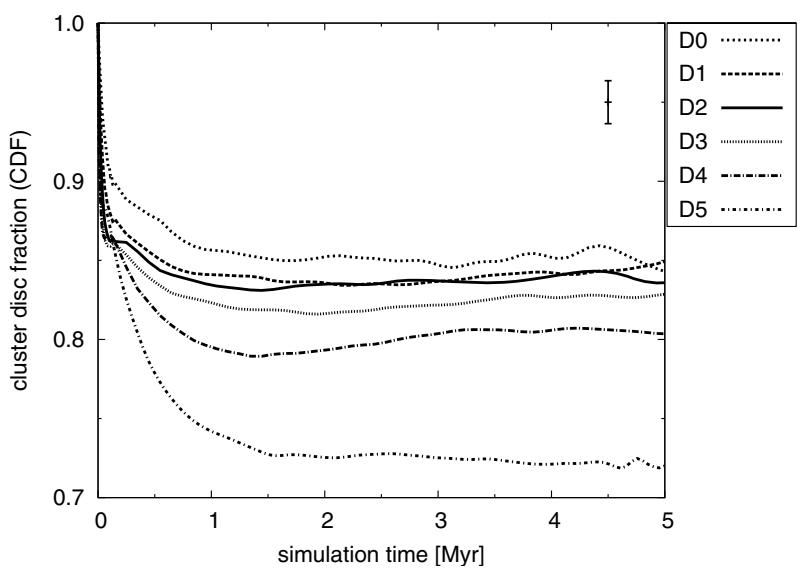

Fig. 7. Time evolution of the cluster disc fraction of the density-scaled models, not restricted to parabolic encounters, for a region of the size of the Trapezium Cluster $\left(R_{\mathrm{TC}}=0.3 \mathrm{pc}\right)$. The curves have been smoothed by Bezier curves to avoid intersecting lines. Here initially equal disc sizes have been assumed. From top to bottom the cluster models D0D5 are marked by a short-dashed, long-dashed, solid, dotted, dot-longdashed, and dot-short-dashed line, respectively. The typical error bar is indicated in the upper right.

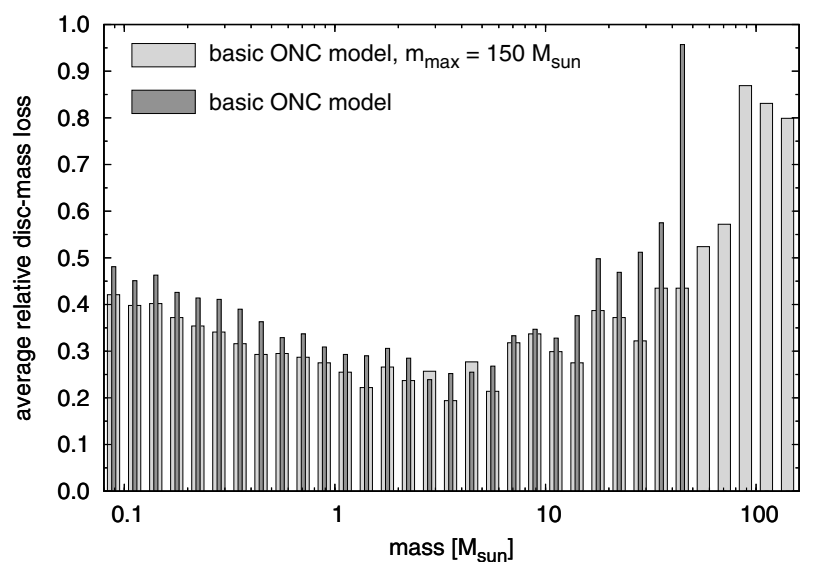

Fig. 8. Average relative disc-mass loss at $1 \mathrm{Myr}$ for the Trapezium Cluster as a function of the stellar mass for initially equal disc sizes. The standard ONC model with a stellar upper mass limit of $50 M_{\odot}$ (dark grey bars) is compared to a simulation of the same model with an upper mass limit of $150 M_{\odot}$ (light grey bars).

shows the average relative disc-mass loss as a function of the stellar mass for the standard ONC model with a stellar upper mass limit of $50 M_{\odot}$ (dark grey boxes), and the same model with an upper mass limit of $150 M_{\odot}$ (light grey boxes). For masses below $10 M_{\odot}$ the two distributions are quantitatively in good agreement.

In the range of $\sim 10-50 M_{\odot}$ the average disc-mass loss of the $50 M_{\odot}$-limit model is significantly higher. This is to be expected because stars in this mass range can act as additional strong gravitational foci in the presence of a $50 M_{\odot}$ star, while their effect is largely reduced if a $150 M_{\odot}$ star is gravitationally dominating. The most massive stars in the range $80-150 M_{\odot}$ show, as expected, the largest average relative disc-mass loss. That it is somewhat lower than the one of the most massive star of the $50 M_{\odot}$-limit model agrees with the stronger gravitational attraction of their disc, leading on average to a reduced disc-mass loss per encounter. However, since the highest mass bin is only populated by nine stars, any further conclusions about the average relative disc-mass loss of the most massive stars would be highly speculative.

\section{Comparison of numerical results and analytical estimates}

In this section the numerical results will be compared to analytical estimates. The treatment of encounters involves one important time scale, the collision time $t_{\text {coll }}$ (Binney \& Tremaine 1987, Eq. (8-123)),

$t_{\mathrm{coll}}=\left[16 \sqrt{\pi} \rho \sigma r_{\star}^{2}\left(1+\frac{G m_{\star}}{2 \sigma^{2} r_{\star}}\right)\right]^{-1}$.

Here the inverse of the collision time will be introduced as the encounter rate, $f_{\text {enc }} \equiv t_{\text {coll }}^{-1}$. Using the escape velocity $v_{\star}$ from the stellar surface,

$v_{\star}=\sqrt{\frac{2 G m_{\star}}{r_{\star}}}$,

the encounter rate can be written as

$f_{\text {enc }}=16 \sqrt{\pi} \rho \sigma r_{\star}^{2}\left(1+\frac{v_{\star}^{2}}{4 \sigma^{2}}\right)$,

where $G$ denotes the gravitational constant, $m_{\star}$ and $r_{\star}$ the stellar mass and radius, and $\rho$ and $\sigma$ the density and velocity dispersion of the star cluster. Below, the stellar radius $r_{\star}$ will be replaced by the "typical interaction radius" $r_{\mathrm{enc}}$, which means the radius at which the star is subject to a significant (but still frequent) perturbation that can potentially remove some fraction of the disc-mass. Equation (12) will be evaluated for three different stellar masses, representing stellar mass groups of low-, intermediate- and high-mass stars. Appropriate typical interaction radii have been taken from Table 3 of Olczak et al. (2006). The set of masses $m_{\star}$, radii $r_{\text {enc }}$, and "encounter escape speeds" $v_{\text {enc }}$ resulting from Eq. (11) is shown in Table 2. The last column contains the "gravitational focusing parameter" $\gamma_{\mathrm{enc}}$, an approximation parameter defined as the power of ten best representing $v_{\text {enc }}^{2}$,

$\gamma_{\mathrm{enc}} \equiv 10^{\left\lfloor\log v_{\mathrm{enc}}^{2}+1 / 2\right\rfloor}$,

where $\lfloor x\rfloor$ denotes the floor function ${ }^{1}$ of $x$. For clarity, massdependent quantities, $x_{\text {enc }}$, will be explicitly denoted as functions of mass, $x_{\text {enc }}\left(m_{\star}\right)$, below.

Because the model D2/S2 represents the standard ONC model, which has been intensively studied, the calculations will be adapted to this model. All quantities related to this model will be denoted accordingly by a " 0 " as subscript. Adopting the initial velocity dispersion of the model D2/S2, $\sigma_{0} \approx 2.3 \mathrm{~km} \mathrm{~s}^{-1} \approx$ $0.5 \mathrm{AU} / \mathrm{yr}$, using

$4 \sigma^{2}=4 \sigma_{0}^{2}\left(\frac{\sigma}{\sigma_{0}}\right)^{2} \approx\left(\frac{\sigma}{\sigma_{0}}\right)^{2} \frac{\mathrm{AU}^{2}}{\mathrm{yr}^{2}}$,

and the numbers given in Table 2, Eq. (12) can be simplified to

$f_{\mathrm{enc}}=16 \sqrt{\pi} \rho \sigma r_{\mathrm{enc}}^{2}\left(m_{\star}\right)\left[1+\gamma_{\mathrm{enc}}\left(m_{\star}\right)\left(\frac{\sigma_{0}}{\sigma}\right)^{2}\right]$.

An even more compact representation is achieved by considering the scaling properties of the two families of models: as can be derived from Eqs. (4) and (6), the scaling relations for the density-scaled models are $\rho \propto N$ and $\sigma \propto \sqrt{N}$, while $\rho=$ const and $\sigma=$ const is found for the size-scaled models. Using these relations, transforming $r_{\mathrm{enc}}\left(m_{\star}\right)$ to $\gamma_{\mathrm{enc}}\left(m_{\star}\right)$ via

1 The floor function $\lfloor x\rfloor$ gives the largest integer less than or equal to $x$. 
Table 3. Approximate relative encounters rates.

\begin{tabular}{llllllll}
\hline \hline $\begin{array}{l}\text { Family of models } \\
\text { cluster model }\end{array}$ & \multicolumn{4}{c}{ Density-scaled } & \multicolumn{3}{c}{ Size-scaled } \\
\hline low mass & $1 / 5$ & $2 / 5$ & 1 & 3 & 7 & 21 & 1 \\
intermediate mass & $1 / 2$ & 1 & 2 & 4 & 9 & 23 & 2 \\
high mass & 5 & 7 & 10 & 15 & 25 & 44 & 10 \\
\hline
\end{tabular}

Eqs. (11) and (13), and adapting the encounter rate to the model $\mathrm{D} 2 / \mathrm{S} 2, f_{\text {enc }}^{\text {norm }} \equiv f_{\text {enc }} / f_{\text {enc }, 0}$, one obtains

$$
\begin{aligned}
f_{\mathrm{enc}}^{\mathrm{norm}} & =\left(\frac{m_{\star}}{m_{\star, 0}} \frac{\gamma_{\mathrm{enc}}\left(m_{\star, 0}\right)}{\gamma_{\mathrm{enc}}\left(m_{\star}\right)}\right)^{2} \\
& \times\left\{\begin{array}{cc}
\left(\frac{\rho}{\rho_{0}}\right)^{3 / 2} \frac{1+\left(\frac{\rho}{\rho_{0}}\right)^{-1} \gamma_{\mathrm{enc}}\left(m_{\star}\right)}{1+\gamma_{\mathrm{enc}}\left(m_{\star, 0}\right)} & \text { D-models, } \\
\frac{1+\gamma_{\mathrm{enc}}\left(m_{\star}\right)}{1+\gamma_{\mathrm{enc}}\left(m_{\star, 0}\right)} & \text { S-models. }
\end{array}\right.
\end{aligned}
$$

The derived relation for the adapted encounter rate predicts very different scaling relations for the two families of cluster models. Density-scaled models are expected to show large variations of the number of encounters, with a strong dependency on the density for low-mass stars, i.e. when $\gamma_{\mathrm{enc}}\left(m_{\star}\right) \ll 1$. In contrast, the encounter rate for the size-scaled models is expected to vary only for the different mass groups, but not among different models. For a better overview of the scaling in terms of numbers, Table 3 lists approximated relative encounter rates $f_{\text {enc }}^{\text {norm }}$, adapted to the low-mass group of the model D2/S2. Table 3 demonstrates that the gravitational focusing parameter plays an important role for the massive stars. The encounter rates increase dramatically by roughly one order of magnitude from the low- and intermediatemass stars to the high-mass stars for the model D2/S2. This finding agrees well with the number of encounters of the ONC model found earlier (cf. Pfalzner et al. 2006).

In summary, what one would expect from the numerical simulations of the density-scaled models is a steep increase of the encounter rate $\propto \rho^{3 / 2}$ in the case of the low-mass stars and a considerably shallower dependency $\propto \rho^{1 / 2}$ for the high-mass stars. For low densities, corresponding to low particle numbers, one would expect that the high-mass stars dominate the encounter rate via gravitational focusing, favouring an overall scaling $\propto \rho^{1 / 2}$. In contrast, the size-scaled models should produce very similar results in terms of encounter rate, regardless of the specific cluster model.

These expectations agree well with the results from the numerical simulations. We demonstrate this in Fig. 9 by the average encounter rate of stars of all masses and of the three mass groups, respectively, adapted in each case to the model D2/S2. Figure 9a shows that the encounter rates of the cluster models D0-D2 are scaling roughly as $N^{1 / 2}$. For higher particle numbers the distribution becomes more complex. Here the high-mass stars show a trend of a decreasing encounter rate with the particle number. This feature accounts for the decreasing importance of the highmass stars as gravitational foci (for the lower mass stars) and is a consequence of the decreasing ratio of the mass of the most massive star and the cluster mass. Accordingly, the distribution of the encounter rate tends towards the analytical limit of $N^{3 / 2}$ for low-mass stars, representing the more frequent interaction of low-mass stars among themselves in the models D3-D5.

Figure $9 \mathrm{~b}$ shows that the size-scaled models are of the same importance in their environmental effect on protoplanetary discs. In the case of low- and intermediate mass stars the presented encounter rates, adapted to the model S2, agree well with the analytical estimate, which predicts a constant distribution as a function of particle number. In contrast, the adapted encounter rate of the high-mass stars decreases with an increasing particle number. This trend shows that the high-mass stars, similar to the finding for the density-scaled models, become less important as gravitational foci for the low-mass stars in clusters with larger stellar populations.

\section{Conclusion and discussion}

The influence of different cluster environments on the encounterinduced disc-mass loss has been investigated by scaling the size, density and stellar number of the basic dynamical model of the ONC. The results can be summarised as follows:

1. The disc-mass loss increases with cluster density but remains unaffected by the size of the stellar population.

2. The density of the ONC itself marks a threshold:

(a) in less dense and less massive clusters it is the massive stars that dominate the encounter-induced disc-mass loss by a gravitational focusing of low-mass stars, whereas

(b) in denser and more massive clusters the interactions of low-mass stars with equal-mass perturbers play the major role for the removal of disc mass.

3. In clusters four times sparser than the ONC the effect of encounters is still apparent.

These results from numerical simulations are well confirmed via observations. It is widely known that the disc frequency of even very young clusters (e.g. NGC 2024) is significantly below 100\% (Haisch et al. 2001; Hillenbrand 2005). This implies a very short time-scale for the disc destruction of a part of the cluster population. In fact, stellar interactions are a potential candidate for a very rapid physical process, and thus the encounter-induced disc-mass loss is potentially a vital mechanism for disc destruction at the earliest stages of cluster evolution. Observational evidence for the role of this mechanism has also been provided for the $\sim 1$ Myr old Orion Nebula Cluster (Olczak et al. 2008). Moreover, observations of NGC 2024, ONC, and NGC 3603 also confirm the correlation of decreasing cluster disc fraction (CDF) with increasing cluster density and even provide evidence for a critical density $\rho_{\text {crit }} \approx \rho_{\text {ONC }}$. A compilation of observational data shows that these similarly aged ( $\sim 1 \mathrm{Myr})$ clusters with peak densities of roughly $10^{3} \mathrm{pc}^{-3}$, $10^{4} \mathrm{pc}^{-3}$, and $10^{5} \mathrm{pc}^{-3}$, respectively, show CDFs of $85 \%, 80 \%$, and 40\% (Haisch et al. 2000; Lada et al. 2000; Stolte et al. 2004). The fact that NGC 2024 is sparser than the ONC but has a similar CDF agrees well with our simulations.

Our results have several important implications for the general picture of star- and cluster formation. Very recently, Pfalzner (2009) has shown that there two cluster sequences exist, evolving in time along pre-defined tracks in the density-radius plane, the "leaky" and the "star-burst" clusters. The simulations performed in the present investigation cover the parameter space of the "leaky" clusters in their embedded stage (see Fig. 10). A comparison with our results shows that at the earliest evolutionary stage leaky clusters have densities above the critical density. Hence in leaky clusters star-disc systems are initially efficiently destroyed via encounters that occur preferentially between low-mass stars. The ONC corresponds to an intermediate stage in the embedded phase of leaky clusters with the transition towards a preferred disc-mass loss of high-mass stars via gravitational focusing of low-mass stars. At the final stage of the embedded phase the encounter-induced disc-mass loss in leaky 
C. Olczak et al.: Stellar interactions in dense and sparse star clusters
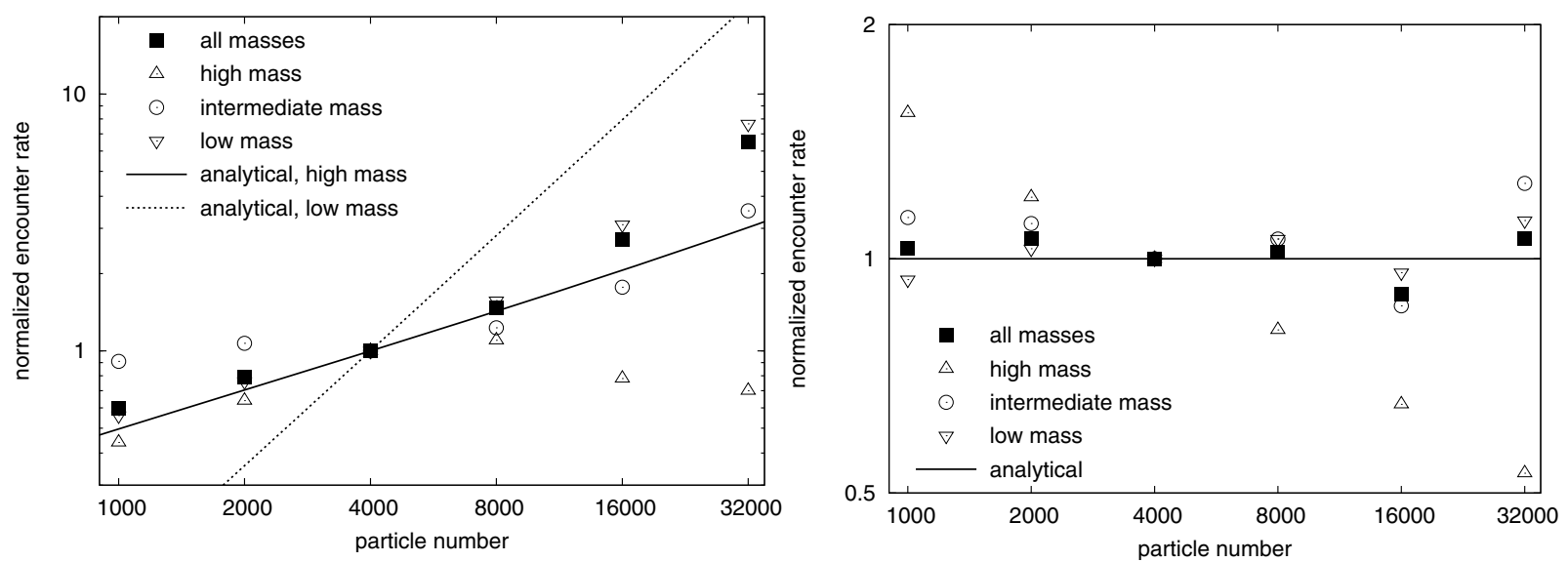

Fig. 9. Normalised encounter rate $f_{\text {enc }}^{\text {norm }}$ from the simulations in comparison with the analytical estimate given by Eq. (15). The filled squares represent all stars in the Trapezium Cluster region $(R=0.3 \mathrm{pc})$, the other open symbols stand for predefined mass groups: high-mass, $m \geq 10 M_{\odot}$ (upward triangles), intermediate-mass, $10 M_{\odot} \geq m \geq 1 M_{\odot}$ (circles), and low-mass stars, $m \leq 1 M_{\odot}$ (downward triangles). The lines depict the analytical estimate of the encounter rate for high-mass (dashed line) and low-mass stars (solid line). The ranges of the mass groups here are different from those in previous figures to account for the mass regimes of the encounter rate presented in Table 2. Left: density-scaled cluster models. Right: size-scaled cluster models (here the analytical estimates of the encounter rate for high-mass and low-mass stars are identical).

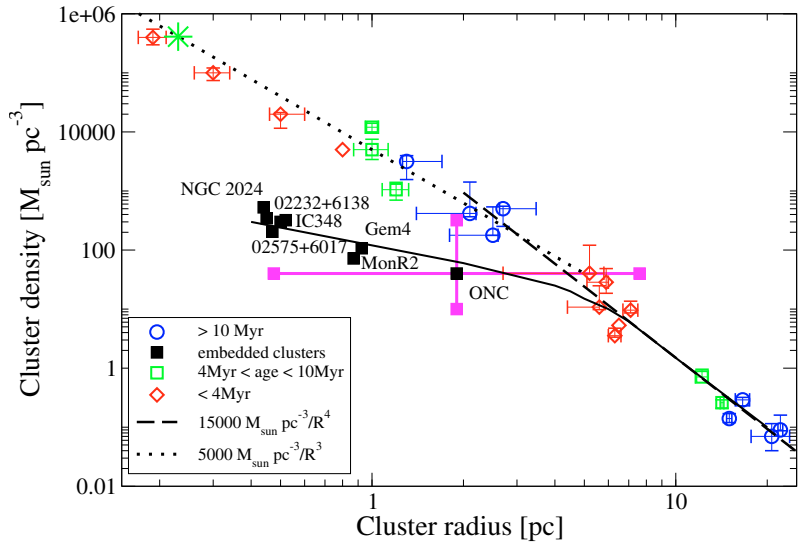

Fig. 10. Cluster density as a function of cluster size for clusters more massive than $10^{3} M_{\odot}$ and embedded clusters with more than 200 observed members from Pfalzner (2009). The parameter space covered by the simulations in this work is indicated by the large pink cross centred on the ONC.

clusters ceases. Gravitational focusing by high-mass stars may still affect single objects, yet at this age is most probably exceeded by other disc-destructive processes like photoevaporation or planet formation. The effects in star-burst clusters would be similar, yet much more pronounced. In case of the Arches cluster one could expect stellar encounters to destroy the discs of most of the low- and high-mass stars in several hundred thousand years. Combining our results with those of Lada \& Lada (2003) that most stars are born in clusters, it becomes evident that a significant fraction of all stars must have been affected by stellar encounters at the early evolutionary stages of their hosting environment.

The application of our results to the dynamics of embedded clusters - though obtained from simulations that do not contain gas - is justified for three reasons:

1. Rather than simulating the evolution of leaky clusters (which would explicitly require the treatment of gas), we use our cluster models to map certain evolutionary stages of the sequence of leaky clusters in terms of "dynamical snapshots". The dynamical effects of these numerical models are then used to estimate the effect of encounters in the observed clusters at their current dynamical state.

2. The effect of gas in an embedded cluster is to lower the frequency of close encounters (due to the smoother cluster potential), yet unless the gas mass is dominating cluster dynamics - as is not the case for the only partially embedded leaky clusters shown in Fig. 10 (e.g. NGC 2024) - the effect is minor.

3. Gas expulsion causes the clusters to expand and thus their density to decrease much faster than in our simulations. Consequently, when mapping our results to the current dynamical state, we underestimate the initial cluster density and thus the effect of encounters in the early evolutionary phases. Hence, our results are least accurate for older clusters, yet well applicable for the young evolutionary stages, where encounters have the most significant effect.

The effect of the encounter-induced disc-mass loss in the early evolution of stellar systems has important implications for the formation and evolution of planets. From our data we conclude that the probability to find planets is highest around intermediate mass stars. While the high initial densities of leaky clusters imply that planets around low-mass stars are expected to be less frequent, hardly any are expected in star-burst clusters. Independent of the cluster environment, planet formation around high-mass stars seems to be completely hindered by interactions with other cluster members.

Acknowledgements. We thank the anonymous referee and the editor for careful reading and very useful comments and suggestions which improved this work. C. Olczak appreciates fruitful discussions with S. Portegies Zwart concerning the analytical estimates and scaling relations. We also thank R. Spurzem for providing the NBODY $6++$ code for the cluster simulations. Simulations were partly performed at the Jülich Supercomputing Centre (JSC), Research Centre Jülich, Project HKU14. We are grateful for the excellent support by the JSC Dispatch team.

\section{Appendix A: Determination of boundaries of mass groups}

Boundaries of mass groups of low-, intermediate- and high-mass stars have been determined individually for different sizes of stellar populations on the basis of the IMF of Kroupa (2001, 
Table A.1. Boundaries of the three mass groups of low-, intermediate-, and high-mass stars of cluster models with 1000, 2000, 4000, 8000, 16000, and 32000 particles.

\begin{tabular}{llllllc}
\hline \hline & 1000 & 2000 & 4000 & 8000 & 16000 & 32000 \\
\hline$m_{0}\left[M_{\odot}\right]$ & $8.00 \times 10^{-2}$ & $8.00 \times 10^{-2}$ & $8.00 \times 10^{-2}$ & $8.00 \times 10^{-2}$ & $8.00 \times 10^{-2}$ & $8.00 \times 10^{-2}$ \\
$m_{1}\left[M_{\odot}\right]$ & $3.30 \times 10^{-1}$ & $3.54 \times 10^{-1}$ & $3.82 \times 10^{-1}$ & $3.95 \times 10^{-1}$ & $4.15 \times 10^{-1}$ & $4.24 \times 10^{-1}$ \\
$m_{2}\left[M_{\odot}\right]$ & 2.94 & 3.78 & 4.95 & 5.58 & 6.61 & 7.14 \\
$m_{3}\left[M_{\odot}\right]$ & $1.47 \times 10^{2}$ & $1.47 \times 10^{2}$ & $1.48 \times 10^{2}$ & $1.48 \times 10^{2}$ & $1.50 \times 10^{2}$ & $1.50 \times 10^{2}$ \\
\hline
\end{tabular}

see also Eq. (1)). The derivation involves the requirement for the three mass ranges to be equidistant in logarithmic space, weighted by the slope of the IMF (of each mass range). The weighting accounts for the steepness of the slope in the highmass regime, which would otherwise cause a very sparsely populated group of high-mass stars.

In the case of a lower mass cutoff at $m_{0}=0.08 M_{\odot}$, and an upper mass limit $m_{3}$, the IMF is characterised by just two different slopes, $\alpha_{1}=1.3$ in the range of $m_{0} \leq m<0.50 M_{\odot}$, and $\alpha_{2}=2.3$ in the range of $0.50 M_{\odot} \leq m \leq m_{3}$. Because the break in the slope of the IMF at the critical mass $m_{c}^{\text {br }}=0.5 M_{\odot}$ does not necessarily coincide with one of the boundaries of the mass ranges, the cases $m_{1}<m_{c}^{\text {br }}$ and $m_{1} \geq m_{c}^{\text {br }}$ have to be differentiated. Though from the theoretical point of view the same differentiation would be required for the higher mass boundary $m_{2}$, this is not relevant for the stellar systems in the focus of the present work. The four mass ranges, $m_{k}, k=0, \ldots, 3$, and the two slopes, $\alpha_{k}, k=1,2$, are then interrelated as follows:

$$
\begin{aligned}
\left(m_{1} \geq\right. & \left.m_{c}^{\mathrm{br}}\right) \wedge\left(m_{2} \geq m_{c}^{\mathrm{br}}\right): \\
& \quad\left(\log m_{1}-\log m_{c}^{\mathrm{br}}\right) \alpha_{2}^{-1}+\left(\log m_{c}^{\mathrm{br}}-\log m_{0}\right) \alpha_{1}^{-1} \\
\equiv & \left(\log m_{2}-\log m_{1}\right) \alpha_{2}^{-1} \\
\equiv & \left(\log m_{3}-\log m_{2}\right) \alpha_{2}^{-1}, \\
\left(m_{1}<\right. & \left.m_{c}^{\mathrm{br}}\right) \wedge\left(m_{2} \geq m_{c}^{\mathrm{br}}\right): \\
& \left(\log m_{1}-\log m_{0}\right) \alpha_{1}^{-1} \\
\equiv & \left(\log m_{2}-\log m_{c}^{\mathrm{br}}\right) \alpha_{2}^{-1}+\left(\log m_{c}^{\mathrm{br}}-\log m_{1}\right) \alpha_{1}^{-1} \\
\equiv & \left(\log m_{3}-\log m_{2}\right) \alpha_{2}^{-1} .
\end{aligned}
$$

Solving these equations, and substituting

$$
\begin{aligned}
\alpha_{12} & \equiv \alpha_{1} \alpha_{2}^{-1}, \\
\alpha_{21} & \equiv \alpha_{2} \alpha_{1}^{-1}, \\
\Gamma_{12} & \equiv 1-\alpha_{12}, \\
\Gamma_{21} & \equiv 1-\alpha_{21},
\end{aligned}
$$

one obtains

$$
\begin{aligned}
& \left(m_{1} \geq m_{c}^{\mathrm{br}}\right) \wedge\left(m_{2} \geq m_{c}^{\mathrm{br}}\right): \\
& \log m_{1}=\frac{1}{3}\left[\log m_{3}+2 \Gamma_{21} \log m_{c}^{\mathrm{br}}+2 \alpha_{21} \log m_{0}\right], \\
& \log m_{2}=\frac{1}{3}\left[2 \log m_{3}+\Gamma_{21} \log m_{c}^{\mathrm{br}}+\alpha_{21} \log m_{0}\right], \\
& \left(m_{1}<m_{c}^{\mathrm{br}}\right) \wedge\left(m_{2} \geq m_{c}^{\mathrm{br}}\right): \\
& \log m_{1}=\frac{1}{3}\left[\alpha_{12} \log m_{3}+\Gamma_{12} \log m_{c}^{\mathrm{br}}+2 \log m_{0}\right] \\
& \log m_{2}=\frac{1}{3}\left[2 \log m_{3}+\Gamma_{21} \log m_{c}^{\mathrm{br}}+\alpha_{21} \log m_{0}\right]
\end{aligned}
$$

Page 10 of 11
The choice of the appropriate solution is determined by the upper mass limit $m_{3}$. For this purpose the "critical maximum mass" $m_{c}^{\max }$,

$$
m_{c}^{\max }=\log ^{-1}\left[\left(1+2 \alpha_{12}\right) \log m_{c}^{\mathrm{br}}-2 \alpha_{12} \log m_{0}\right]
$$

is estimated from Eq. (A) and $m_{1} \equiv m_{c}^{\text {br }}$. Consequently, the following relations apply:

$$
\begin{array}{lll}
m_{3}<m_{c}^{\max } & \Longrightarrow & m_{1} \geq m_{c}^{\mathrm{br}}, \\
m_{3} \geq m_{c}^{\max } & \Longrightarrow & m_{1}<m_{c}^{\mathrm{br}} .
\end{array}
$$

With the given values of the parameters $m_{0}, \alpha_{1}$, and $\alpha_{2}$ one finds $m_{c}^{\max } \approx 3.97 M_{\odot}$.

The derived mass boundaries, $m_{k}, k=0, \ldots, 3$, for each cluster of the families of models are presented in Table A.1.

\section{References}

Aarseth, S. 2003, Gravitational N-body Simulations (Cambridge: Cambridge University Press), 430

Adams, F. C., Proszkow, E. M., Fatuzzo, M., et al. 2006, ApJ, 641, 504 Alexander, R. D., Clarke, C. J., \& Pringle, J. E. 2005, MNRAS, 358, 283 Alexander, R. D., Clarke, C. J., \& Pringle, J. E. 2006, MNRAS, 369, 229 Bally, J., Testi, L., Sargent, A., et al. 1998, AJ, 116, 854

Balog, Z., Muzerolle, J., Rieke, G. H., et al. 2007, ApJ, 660, 1532

Bik, A., Lenorzer, A., Kaper, L., et al. 2003, A\&A, 404, 249

Binney, J., \& Tremaine, S. 1987, Galactic dynamics (Princeton, NJ: Princeton University Press), 747

Clarke, C. J., \& Pringle, J. E. 1993, MNRAS, 261, 190

Clarke, C. J., Bonnell, I. A., \& Hillenbrand, L. A. 2000, Protostars and Planets IV, 151

Clarke, C. J., Gendrin, A., \& Sotomayor, M. 2001, MNRAS, 328, 485

Currie, T., Kenyon, S. J., Balog, Z., et al. 2008, ApJ, 672, 558

de Pree, C. G., Nysewander, M. C., \& Goss, W. M. 1999, AJ, 117, 2902

Drake, J. J., Ercolano, B., Flaccomio, E., et al. 2009, ApJ, 699, L35

Ercolano, B., Drake, J. J., Raymond, J. C., et al. 2008, ApJ, 688, 398

Falgarone, E., \& Phillips, T. G. 1990, ApJ, 359, 344

Figer, D. F. 2005, Nature, 434, 192

Gorti, U., \& Hollenbach, D. 2009, ApJ, 690, 1539

Haisch, K. E., Lada, E. A., \& Lada, C. J. 2000, AJ, 120, 1396

Haisch, Jr., K. E., Lada, E. A., \& Lada, C. J. 2001, ApJ, 553, L153

Hall, S. M. 1997, MNRAS, 287, 148

Hall, S. M., Clarke, C. J., \& Pringle, J. E. 1996, MNRAS, 278, 303

Heller, C. H. 1993, ApJ, 408, 337

Heller, C. H. 1995, ApJ, 455, 252

Hillenbrand, L. A. 1997, AJ, 113, 1733

Hillenbrand, L. A. 2002 [arXiv: 0210520]

Hillenbrand, L. A. 2005 [arXiv:0511083]

Hillenbrand, L. A., \& Carpenter, J. M. 2000, ApJ, 540, 236

Hillenbrand, L. A., \& Hartmann, L. W. 1998, ApJ, 492, 540

Hillenbrand, L. A., Strom, S. E., Calvet, N., et al. 1998, AJ, 116, 1816

Hollenbach, D. J., Yorke, H. W., \& Johnstone, D. 2000, Protostars and Planets IV, 401

Johnstone, D., Hollenbach, D., \& Bally, J. 1998, ApJ, 499, 758

Johnstone, D., Matsuyama, I., McCarthy, I. G., et al. 2004, in Rev. Mex. Astron.

Astrofis. Conf. Ser. 22, ed. G. Garcia-Segura, G. Tenorio-Tagle, J. Franco, \&

H. W. Yorke, 38

Kley, W., Papaloizou, J. C. B., \& Ogilvie, G. I. 2008, A\&A, 487, 671

Koen, C. 2006, MNRAS, 365, 590 
C. Olczak et al.: Stellar interactions in dense and sparse star clusters

Kroupa, P. 2001, MNRAS, 322, 231

Kroupa, P. 2002, Science, 295, 82

Lada, C. J., \& Lada, E. A. 2003, ARA\&A, 41, 57

Lada, C. J., Muench, A. A., Haisch, Jr., K. E., et al. 2000, AJ, 120, 3162

Larson, R. B. 2003, in Galactic Star Formation Across the Stellar Mass Spectrum, ed. J. M. De Buizer, \& N. S. van der Bliek, ASP Conf. Ser., 287, 65

Liu, W. M., Meyer, M. R., Cotera, A. S., et al. 2003, AJ, 126, 1665

Luhman, K. L., Rieke, G. H., Young, E. T., et al. 2000, ApJ, 540, 1016

Maíz Apellániz, J., Walborn, N. R., Morrell, N. I., Niemela, V. S., \& Nelan, E. P. 2007, ApJ, 660, 1480

Mann, R. K., \& Williams, J. P. 2009, ApJ, 699, L55

Maschberger, T., \& Clarke, C. J. 2008, MNRAS, 391, 711

Matsuyama, I., Johnstone, D., \& Hartmann, L. 2003, ApJ, 582, 893

McCaughrean, M., Zinnecker, H., Andersen, M., Meeus, G., \& Lodieu, N. 2002, The Messenger, 109, 28

Miesch, M. S., \& Scalo, J. M. 1995, ApJ, 450, L27

Moeckel, N., \& Bally, J. 2006, ApJ, 653, 437

Moeckel, N., \& Bally, J. 2007a, ApJ, 661, L183

Moeckel, N., \& Bally, J. 2007b, ApJ, 656, 275

Muench, A. A., Lada, E. A., Lada, C. J., et al. 2002, ApJ, 573, 366

Oey, M. S., \& Clarke, C. J. 2005, ApJ, 620, L43

Olczak, C., Pfalzner, S., \& Eckart, A. 2008, A\&A, 488, 191
Olczak, C., Pfalzner, S., \& Spurzem, R. 2006, ApJ, 642, 1140

Ostriker, E. C. 1994, ApJ, 424, 292

Palla, F., \& Stahler, S. W. 2000, ApJ, 540, 255

Pfalzner, S. 2004, ApJ, 602, 356

Pfalzner, S. 2006, ApJ, 652, L129

Pfalzner, S. 2009, A\&A, 498, L37

Pfalzner, S., \& Olczak, C. 2007a, A\&A, 462, 193

Pfalzner, S., \& Olczak, C. 2007b, A\&A, 475, 875

Pfalzner, S., Umbreit, S., \& Henning, T. 2005, ApJ, 629, 526

Pfalzner, S., Olczak, C., \& Eckart, A. 2006, A\&A, 454, 811

Pfalzner, S., Tackenberg, J., \& Steinhausen, M. 2008, A\&A, 487, L45

Scally, A., \& Clarke, C. 2001, MNRAS, 325, 449

Scally, A., Clarke, C., \& McCaughrean, M. J. 2005, MNRAS, 358, 742

Sicilia-Aguilar, A., Hartmann, L., Calvet, N., et al. 2006, ApJ, 638, 897

Slesnick, C. L., Hillenbrand, L. A., \& Carpenter, J. M. 2004, ApJ, 610, 1045

Spurzem, R. 1999, J. Comput. Appl. Math., 109, 407

Stolte, A., Brandner, W., Brandl, B., Zinnecker, H., \& Grebel, E. K. 2004, AJ, 128,765

Stolte, A., Brandner, W., Brandl, B., et al. 2006, AJ, 132, 253

Störzer, H., \& Hollenbach, D. 1999, ApJ, 515, 669

Weidner, C., \& Kroupa, P. 2006, MNRAS, 365, 1333

Williams, J. P., Andrews, S. M., \& Wilner, D. J. 2005, ApJ, 634, 495

Zinnecker, H., \& Yorke, H. W. 2007, ARA\&A, 45, 481 